\title{
Dynamical strain measurement by IRT
}

\author{
by J. JOUGLAR, M. MERGUI, P. L. VUILLERMOZ
}

Laboratoire de Physique de la Matière (URM C 5511), INSA LYON, 20 Av. A. Einstein, 69621 VILLEURBANNE Cedex, FRANCE. Tel: 72.43.80.67 Fax: 72.43.85.31

\begin{abstract}
We have developped a signal processing technique allowing to measure by IRT the strain distribution on the surface of a sample submitted to a sinusoidal bending stress. Line mode operation of the scanner allows time and memory saving. A sliding-average filtering gives a thermal resolution of about $1 \mathrm{~m}^{\circ} \mathrm{C}$. The dynamic signal is extracted from the frequency spectrum by the averaged periodogram technique, without any lock-in system. The precision of the result is discussed by comparison with strain gauge measurements.
\end{abstract}

\section{Introduction}

In order to foresee the occurrence of defects which may be damaging for a structure, it is useful to know the strains and stresses in this structure. It is not possible to measure internal stresses, but surface strain measurements give access to superficial stresses, generally the most dangerous, which constitute an image of internal ones. The thermoelasticity laws relate dynamical stresses to the surface temperature on a sample submitted to a periodical bending stress. Infrared thermographiy (IRT) is then a useful tool for strain characterization. But the temperature variations to be measured are smaller than the thermal resolution of the camera, so signal processing is necessary. Several solutions have been proposed and some commercial realisations are now available [1-5], but most of them use a lock-in step which requires a reference signal rigorously synchronised with the measurement. This paper describes a signal processing technique using non-linear filtering and signal extraction from the Fourier Transform. Line mode operating of the camera allows time and computer memory saving in-order to make possible measurements on evolving systems. The performances of this technique have been evaluated by a systematic study of noise before and after treatment, and by comparison with strain gauge measurements.

\section{Experimental configuration}

The sample is a PVC parallelepiped $\left(380^{\star} 50^{\star} 15 \mathrm{~mm}^{3}\right)$ fixed at the bottom and binded at the other end to a vibrating pot. The material has been chosen for its opacity to infrared radiation and its low thermal conductivity. The surface emissivity has been artificially made homogeneous and close to 1 by grinding and $\mathrm{MgO}$ layer deposition.

The scanner is an AGEMA 880 Short Wave with a $12^{\circ}$ lens, associated to a PC 80386 DX33 with a $700 \mathrm{M}$ hard disk. The signal is digitized on 12 bits.

Strain gauges glued on the sample will allow to calibrate our results by a classical technique. The vibrating pot will be operated at $30 \mathrm{~Hz}$, near the first resonance frequency of the sample. The applied force is comprised between 2 and 10 Newton, giving a maximum strain of 150 to $650 \mu \mathrm{m} / \mathrm{m}$. The accuracy of strain gauge measurements is $5.5 \%$.

\section{Thermoelasticity}

When a periodical stress is applied to an isotropic solid sample, thermomechanical phenomena may be described $[6,7]$ by the equation (1)

$$
\rho \mathrm{C} \dot{\mathrm{T}}=\mathrm{k} \Delta \mathrm{T}+\sigma: \delta^{\mathrm{p}}+\mathrm{T}\left[\frac{\partial \sigma}{\partial \mathrm{T}}: \delta^{\bullet}\right]
$$


( $\rho:$ specific mass ; $C$ : specific heat $; k$ : thermal conductivity $; \sigma:$ stress tensor $; \delta^{p}$ : elastic strain tensor; $T$ : temperature ).

The first term in the right part of equation (1) is a heat diffusion term; it will be neglected if $k$ is small. The second term, due to inelastic dissipation, leads to a slowly increasing temperature component $T_{1}$. The third term is the thermoelastic coupling term : it produces a periodic variation $T_{2}$ of the temperature around the $T_{1}$ value.

Under some restrictive hypotheses [2] (small thickness for the sample, small strains, temperature near ambient temperature), the solution of equation (1) is given by

$$
\sigma=-\frac{\rho \mathrm{C}}{\alpha} \Delta \mathrm{T}_{2}
$$

( $\alpha$ : linear expansion coefficient)

\section{Noise characterization}

There are several sources of random noise in the measurement apparatus : transducer, electronic circuits, digitization. These different noises, either correlated or not, are mixed in the system and may be defined by a total variance $\sigma_{T}^{2}$. There are also some parasitic signals (emissivity inhomogeneities, reflexions, non uniformity of the scanner response).

The characteristics of the noise have been measured in the following way. After stabilization during $30 \mathrm{mn}$, the sample surface temperature is uniform and stable. Three thousand images are recorded, then averaged pixel by pixel : the random noise is practically suppressed. This average image is substracted from a single image : the parasitic signals disappear and we obtain a noise picture. A statistical study of this noise shows a gaussian shape with a standard deviation of 132 binary levels, which leads to a thermal resolution of $0.17^{\circ} \mathrm{C}$. A frequency analysis by Fourier Transform shows an increasing noise density in the 0 to $2 \mathrm{kHz}$ range. Low frequency filtering will then be needed.

\section{Line mode acquisition}

This acquisition mode has several advantages with regard to the usual frame mode : higher speed, no time wasting by frame return, possibility to study only a limited number of lines corresponding to the interesting part of the field of view. We have chosen to record $N$ times the same line by monitoring the vertical scanning motor through an RS 232 interface. The signal is then processed (cf. $\S 6,7$ ) and another line is selected. The useful line length is $320 \mu \mathrm{s}$ and the sampling frequency is $400 \mathrm{kHz}$, therefore 128 pixels are digitized on each line. It would be interesting to store all the lines of the image and to perform signal processing afterwards; this method would be better when the signal is quickly varying, but it needs a great capacity computer memory (100 Mb).

\section{Filtering}

The study of the noise has shown the necessity of rigorous filtering, well suited to the system characteristics (spatial resolution, thermal resolution, spectral response...). Several filters have been tested, the best results have been obtained with a sliding average filter. If $x\left(k T_{e}\right)$ is the signal measured on the $k^{\text {th }}$ pixel $\left(T_{\theta}\right.$ : sampling frequency $\left.=400 \mathrm{kHz}\right)$, the filter will attribute to this pixel the value $y\left(k T_{e}\right)$ according to equation (3)

$$
y\left(k T_{\theta}\right)=(1 / n) \sum_{l=k-p}^{k+p} x\left(I T_{\theta}\right)
$$

with $n=2 p+1$.

For a given position of the window of width $\theta\left(\theta=n T_{\theta}\right)$, we obtain $N$ average values of the thermosignal ( $N=$ number of recordings of the same line ). This means that we sample the signal at the line frequency $(2500 \mathrm{~Hz})$ with a sampling pulse of length $\theta=n T_{e}=n$ * $2.5 \mu \mathrm{s}$. The 
signal spectrum is filtered by $\sin (\pi v \theta) /(\pi v \theta)$ in amplitude and by $-2 \pi v \theta / 2$ in phase $(v=$ frequency).

For an amplitude attenuation lower than $1 \%$ at the highest frequency included in the signal, the filter width $n$ must be lower than 25 pixels. For a phase shift lower than 5 degrees, it must be lower than 9 .

The noise standard deviation $\sigma_{B}$ has been measured for various $n$ values : it decreases as $1 / \sqrt{ } n$ for $n \geq 2$, but is 12 binary levels larger than the theoretical value (obtained with $\sigma_{B}=131$ $B L$ for $n=1$ ). This difference may be due to a redistribution of noise by the filter.

The thermal resolution, calculated after the noise standard deviation, is plotted on figure 1. The resolution is of about $1 \mathrm{~m}^{\circ} \mathrm{C}$ for $\mathrm{n}=20$, and $2.7 \mathrm{~m}^{\circ} \mathrm{C}$ for $\mathrm{n}=2$ ( for $\mathrm{N}=2048$ samples).

\section{Spectral analysis}

In dynamic measurements, the frequency spectrum will allow to separate the strain signal from the noise : a sorting routine will determine the highest amplitude in this spectrum.

If $y_{\theta, k, i}\left(i T_{1}\right)$ is a value of the signal after filtering ( $\theta:$ filter width, $k:$ position of the window on the line, $i$ : number of the measurement $1<i<N, T_{1}$ : line period : $400 \mu \mathrm{s}$ ), the Fourier transform is

$$
Y(v, N)=\sum_{i=0}^{N-1} y_{\theta k i}\left(i T_{1}\right) f\left(i T_{1}\right) e^{-2 j \pi v i}
$$

where $f\left(i T_{1}\right)$ is a window function of width $\Theta=N T_{1}$ due to the fact that the acquisition has been done during the time $\Theta$. The spectral density will be calculated by a non-parametric estimation technique called periodogram

$$
S_{Y Y}(v)=(1 / N)|Y(v, N)|^{2}
$$

(this periodogram is the Fourier transform of the autocorrelation function ). The variance of the error due to the limited time $\Theta$ of acquisition is [9] $\sigma^{2}=2 \alpha / \mathrm{N}$ where $2 \alpha$ is the oversampling factor. Our sampling frequency is $2500 \mathrm{~Hz}$ and we wish to use the frequency range from 0 to $125 \mathrm{~Hz}$, that is $2 \alpha=20$. In this case $\sigma \sim 0.14$ for $N=1024$. Figure 2 shows the signal spectrum in the case of a $30 \mathrm{~Hz}$ excitation, and for two values of the filter width. One notes that for small $n$, the $60 \mathrm{~Hz}$ harmonic is clearly seen.

This simple periodogram technique may be improved by averaging $M$ periodograms, each of them of length $N$; the variance will be reduced $M$ times. For a given value of the product $N M$ which determines the acquisition time, the spectral resolution will be better if $N$ increases, and the variance will decrease if $M$ increases. A compromise must be found, also taking into account the stability of the signal during the measurement.

The stress signal will be found in the spectrum as the component of highest amplitude, but the excitation frequency may not be an integral multiple of the frequency step. In order to overcome this non synchronisation problem, we use a Blackman weighing window according to equation (6)

$$
f_{B}=0.42+0.5 \cos (2 \pi i / N)+0.08 \cos (4 \pi i / N)
$$

\section{Experimental results}

The best parameter values have been chosen after a systematic study of stress measurements in the following conditions : sinusoïdal force of amplitude $7.6 \mathrm{~N}$ and frequency $30 \mathrm{~Hz}$; scanner-sample distance: $1 \mathrm{~m}$; frame scanning blocked on line $\mathrm{n}^{\circ} 8$ corresponding to the position of the extensometry gauges. We shall use the following values : number of line recordings $N=2048$; filter width $n=5$ pixels; number of periodograms $M=10$.

Figure 3 shows two stress profiles along the sample; the upper curve is a theoretical straight line, calibrated on line $n^{\circ} 8$ by strain gauge measurement. The lower plot figures the thermosignal values measured on the same pixel of each line, after treatment with the 
previously determined parameters. The difference in slope is not significant if the low stress values are not taken into account, but a slight error on the slope may be due to the evolution of ambient temperature during the measurement $\left(\sim 0.15^{\circ} \mathrm{C} / 15 \mathrm{~mm}\right)$. The main error $(\sim 15 \%)$ comes probably from the Young modulus value. Figure 4 shows a 3D representation of stress on the whole field of view, obtained by juxtaposition of stress profiles. Isostress lines are clearly seen, which is consistent with the geometry of the system.

We have applied our signal treatment technique to several cases of stress concentration around artificial defects. Figure 5 is a 3D representation of stress profile on a sample with a transverse groove of depth half the sample thickness. The concentration factor, determined by reference to figure 3 , is 2.2 . This value is consistent with the theoretical value 2.3 calculated after [10].

\section{Conclusion}

This signal treatement using line mode acquisition, non linear filtering and periodogram averaging gives stress distributions in good quantitative agreement with a classical extensometry method. It provides a better thermal resolution than a simple frame averaging for the same processing time. The absence of any lock-in step gives a great versatility for there is no need for a reference signal. Line mode recording allows memory saving and faster recording, which is interesting if the thermosignal varies; moreover it is possible to record the signal of only a part of the field of view. At the present time, we cannot measure stresses lower than $0.4 \mathrm{MPa}$, but this can be improved with a computer having a greater memory and a higher processing speed. Finally, the precision of absolute measurements is limited by the knowledge of emissivity, as in any IR technique, and by imperfections of the scanner ( non uniformity of the response in the field of view, drift...); a thorough study of these parameters leads to corrective factors [11].

\section{REFERENCES}

[1] PEDRON (J.P.). - Application de la mesure de contraintes par thermographie infrarouge aux structures automobiles. Revue automobile $n^{\circ} 673$, Mai 1988, p. 82-86.

[2] BREMOND (P.). - Développement d'une instrumentation infrarouge pour l'étude des structures mécaniques. Application à l' étude des extrémités de fissures. Thèse de docteur ingénieur. Université d' Aix-Marseille, 86 p, 1982.

[3] PYE (J.C.) and ADAMS (R.D.). - Detection of damage in fibre reinforced plastics using thermal fields generated during resonant vibration. NDT International, June 1981, p. 86-95.

[4] CHRYSOCHOOS (A.), CHEZEAUX (J.C.) et CAUMON (H.). - Analyse thermomécanique des lois de comportement par thermographie infrarouge. Rev. Phys. Appl., t.24, $n^{\circ} 2,1989, p$. 215-225.

[5] LEVESQUE (P.), LISIECKI (B.), CARON (P.), KUBIN (L.), DEOM (A.) and BALAGEAS (D.). - Infrared thermography of plastic instabilities in a single crystal superalloy. EETI ed. Eurotherm series 27,1992, p. 134-141.

[6] GERMAIN (P.). - Cours de mécanique des milieux continus. Ecole Polytechnique ed., 375 p, 1980.

[7] CHRYSOCHOOS (A.). - Bilan énergétique en élastoplasticité grandes déformations. J. Mécanique Théor. Appl., 1985, vol.4, n5, p. 589-614.

[8] KUNT (M.), BELLANGER (M.), de COULON (F.), GUEGUEN (C.), HASLER (M.), MOREAU (N.) and VETTERLI (M.). - Techniques modemes de traitement numérique des signaux. Lausanne : Presses Polytechniques et Universitaires Romandes,471 p, 1991, vol.1. 
[9] LAND (H.) and POLLACK (H.D.). - Modern spectral estimation. Bell System Technichal Journal, 1992, nº41, p. 1295-1336.

[10] WARREN YOUNG (C.). - Roark's formulas for stress and strain. New York, Mc Graw Hill, $763 p, 1989$.

[11] MERGUI (M.). - Mesure de contraintes dynamiques par thermographie infrarouge. Thèse. Oct. 95. Institut National des Sciences Appliquées de Lyon, $176 \mathrm{p}$.

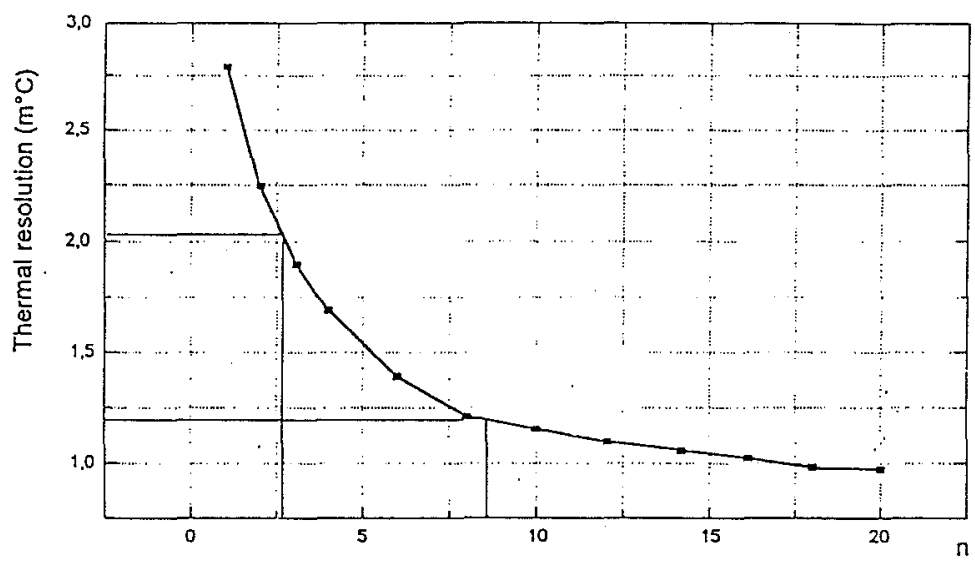

Fig. 1. Thermal resolution vs. filter width $n$ (pixels)
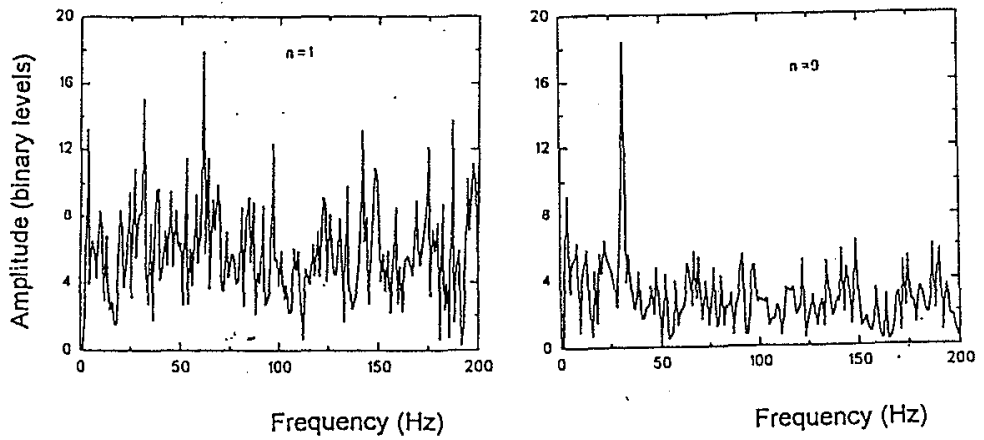

Fig. 2 . Signal spectra for $N=2048$ and for two filter widths : $n=1$ (left), $n=9$ (right) 


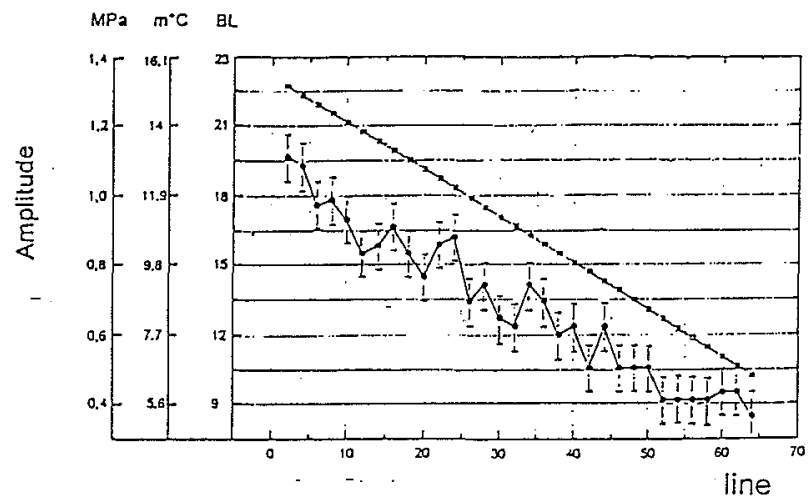

Fig. 3 . Stress profile along the sample

Upper curve : extensometry; lower curve: IR measurements. In abscissa lines are numbered from 1 (near the fixed end of the sample) to 64 (nearer the force application point).

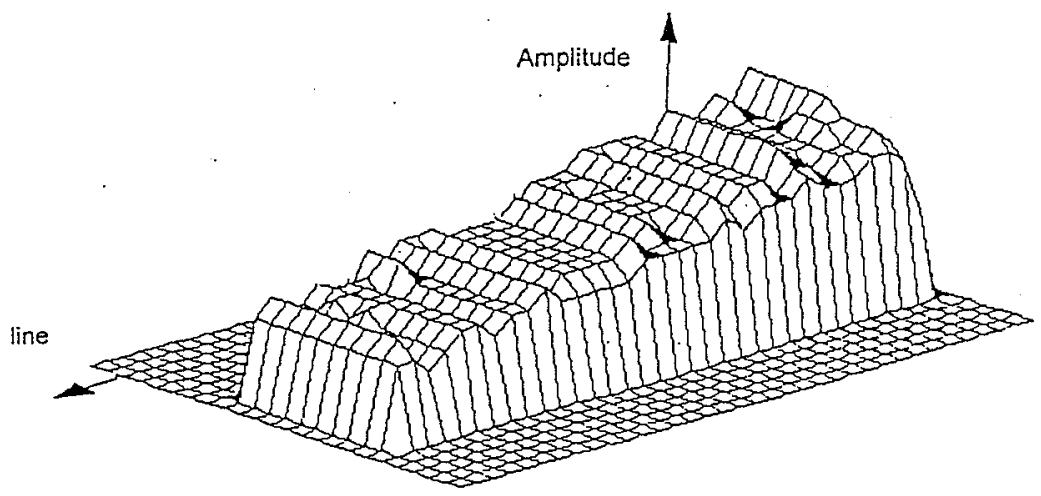

Fig. 4 . 3D stress mapping on the sample

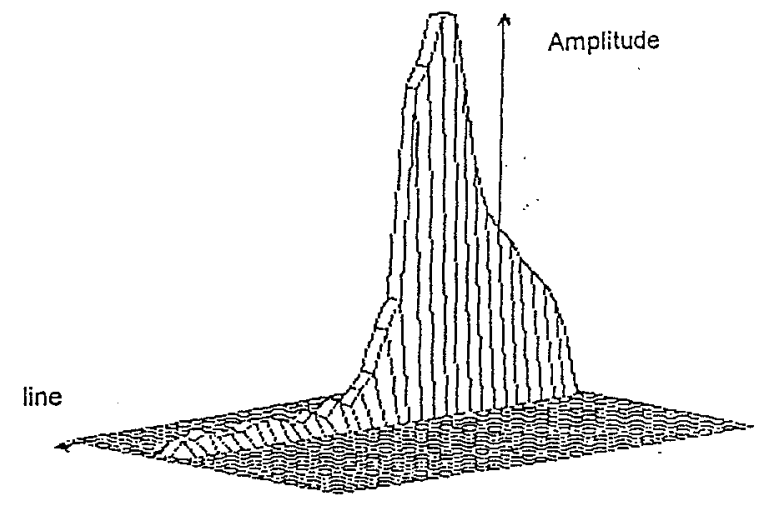

Fig. 5 . 3D stress concentration profile 